\title{
Digital fringe projection for hand surface coordinate variation analysis caused by osteoarthritis
}

\author{
Wan Mokhdzani Wan Nor Haimi', , Cheek Hau $\operatorname{Tan}^{l}$, Vithyacharan Retnasamy ${ }^{l}$, Rajendaran Vairavan ${ }^{l}$, Zaliman Sauli ${ }^{l}$, \\ Nor Roshidah Yusof, Nor Azura Malini Ahmad Hambali ${ }^{1}$, Muhammad Hafiz Ab Aziz ${ }^{1}$ and Ahmad Syahir Ahmad Bakhit ${ }^{l}$
}

${ }^{1}$ School of Microelectronic Engineering, Universiti Malaysia Perlis, Malaysia

\begin{abstract}
Hand osteoarthritis is one of the most common forms of arthritis which impact millions of people worldwide. The disabling problem occurs when the protective cartilage on the boundaries of bones wear off over time. Currently, in order to identify hand osteoarthritis, special instruments namely X-ray scanning and MRI are used for the detection but it also has its limitations such as radiation exposure and can be quite costly. In this work, an optical metrology system based on digital fringe projection which comprises of an LCD projector, CCD camera and a personal computer has been developed to anticipate abnormal growth or deformation on the joints of the hand which are common symptoms of osteoarthritis. The main concept of this optical metrology system is to apply structured light as imaging source for surface change detection. The imaging source utilizes fringe patterns generated by $\mathrm{C}++$ programming and is shifted by 3 phase shifts based on the 3 steps 2 shifts method. Phase wrapping technique and analysis were applied in order to detect the deformation of live subjects. The result has demonstrated a successful method of hand deformation detection based on the pixel tracking differences of a normal and deformed state.
\end{abstract}

\section{Introduction}

Osteoarthritis (OA) is a relatively prevalence musculoskeletal disorder problem which frequently affects the joints in chronic conditions and it is the most common type of arthritis. Hand $\mathrm{OA}$ is a joint degeneration disease due to the failure reparation of joint damage which causes stiffness, pain and impaired movement[1,2]. According to the World Health Organization (WHO), the Department of Chronic Diseases and Health Promotion estimated that $10 \%$ to $15 \%$ of all adults aged over 60 have a certain degree of osteoarthritis, with OA more common among women compared to men[3]. Knees, hips, hands, big toes and spine are common body parts where OA can develop. A population-based analysis based on National Health Examination Survey (NHES) data found that the prevalence of hand OA was $29.5 \%$ among those aged 25 years old and onwards[4]. The probability of getting hand $\mathrm{OA}$ was higher compared to the feet $\mathrm{OA}$ even though both of these body parts has equal numbers of joint. This is due to the cartilage inside the joints of the hand are used more regularly than the feet to cover and protect the end tips of bones where they meet to form a joint.

In general, OA can be characterised by three types of diagnosis which is clinical, pathological and radiological[5]. Pathological diagnosis are carried out in certain focal areas within synovial joints that are located with hypertrophy of bone and thickening capsule and are inspected by x-rays. In the clinical analysis, the OA syndrome can be classified by joint pain, tenderness, restriction of movement, crepitus, occasionally effusion and variety degrees of local inflammation where significant physical symptoms of OA can be seen.

Radiographic diagnosis is executed with X-rays which can detect changes of the interphalangeal joints such as focal narrowing, marginal osteophyte changes and sclerosis. The X-ray utilizes high energy level that allows the beam to penetrate through the body and create an image. Since X-rays applies ionizing radiation, cells can be damaged and develop into cancer. A short duration and small dosage have been know to avoid cells being damaged namely diagnosis such as the mammogram $[6,7]$.

Several other methods of hand OA diagnostic has also been developed throughout the years such as the Magnetic Resonance Imaging (MRI), Micro CT Scanning and ultrasonography. The MRI diagnostic method has long being applied in medical image diagnosis. It applies a magnetic field and pulses of radio wave energy to create image of organs and bones inside the body which also provides different image information compared to X-rays and CT scan. However, there is a limitation to the MRI in terms of low sensitivity, low specificity and also cost ineffectiveness [8].

* Corresponding author: wanmokhdzani@unimap.edu.my 
The Micro-CT scanning method is a non-invasive, whole volume imaging technology that provides high contrast between bone and soft tissues and produces quantitative data on bone stereology[9]. It is the advanced version of $\mathrm{X}$-ray because micro-CT scanning offers higher resolution than a plain $\mathrm{X}$-ray. Although both diagnostic tools use $\mathrm{X}$-rays and ionizing radiation as imaging sources, micro-CT scanning enables one to view in $3 \mathrm{D}$ microscopy.

The Ultrasonography method appears favourable to assess $\mathrm{OA}$ in both clinic and clinical trials. It possesses higher resolutions than conventional radiographs, does not involve any ionising radiation, and allows multiplanar dynamic imaging of joints[10].

The method of hand OA diagnostic proposed in this work is by using the digital fringe projection based on structured light projection. Digital fringe projection has previously been applied on human subjects for analysis on face wrinkles, dental analysis, fingerprint imaging, human skin and wound dimension analysis [11-16]. Based on the literature reviews carried and to the best of the author's knowledge, hand OA detection using digital fringe projection has been quite limited. The digital fringe projection technique applied in this work utilizes the phase shifting method. Human hand were tested whereby two states of the hand were assessed, normal state and deformed state of the hand under the fringe projected image with phase shifting.

\section{Theory}

In this work, the basic $\mathrm{C}++$ language is applied to generate three fringe patterns with different phase. The phase angle is shifted every $2 \pi / 3$ radians respectively to the three different fringe patterns. The three-step technique of phase shifting method is applied to obtain phase map. The mathematical descriptions of these three fringe patterns are as[16]:

$$
\begin{gathered}
I_{1}=A(i, j)+B(i, j) \cos \phi(i, j) \\
I_{2}=A(i, j)+B(i, j) \cos [\phi(i, j)+2 \pi / 3] \\
I_{3}=A(i, j)+B(i, j) \cos [\phi(i, j)+4 \pi / 3]
\end{gathered}
$$

Where $A(i, j)$ is the uniform background intensity, $B(i, j)$ is the amplitude of sinusoidal waveform and $\phi(i, j)$ is the phase angle at the point $(i, j)$. The three equations are also known as the resultant intensity patterns.

The phase wrapped mapping can be obtained by evaluating $\phi(i, j)$ simultaneously with Equation 1, 2 and 3 which leads t0 Equation 4:

$$
\phi(i, j)=\tan ^{-1}\left[\sqrt{ } 3\left[I_{3}(i, j)-I_{2}(i, j)\right] / 2 I_{1}(i, j)-I_{3}(i, j)-I_{2}(i, j)\right]
$$

The significance of $\phi$ given by equation (4) is within $\pm \pi$. Nevertheless, by examining the signs of the numerator and denominator, the significance of $\phi$ between 0 to $2 \pi$ can be resulted [17].

\section{Methodology}

\subsection{Experimental Setup}

The general fringe projection system setup used for the hand deformation inspection is shown in Figure 1. A standard LCD projector ((Panasonic PT-LW80NTEA) is utilized to project computer generated fringe patterns and images are recorded with a CCD camera(monochrome (AVT Marlin F-146C) 1392 x 1040 resolution). The LCD projector and digital CCD camera are mounted on top of the experimental surface under test. The sinusoidal fringe patterns were programmed using $\mathrm{C}++$ in Microsoft Visual Studio 2010. The hand used as the test subject was placed on the reference plane and fringe visibility was best observed at a height of $490 \mathrm{~mm}$ above the hand sample after a series of fringe visibility analysis based on camera distance from the hand sample.

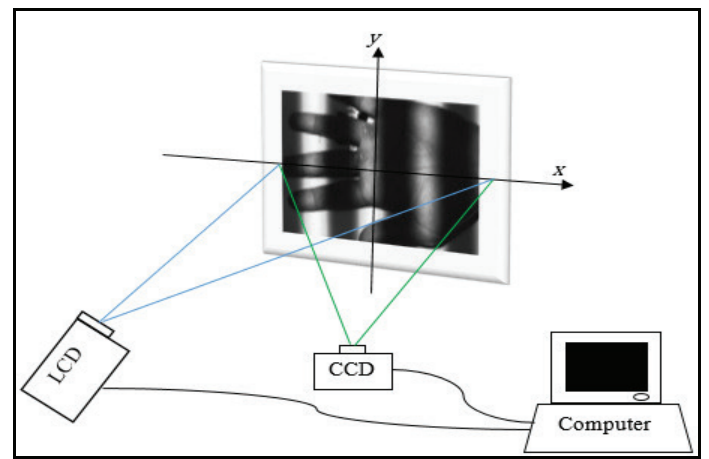

Fig. 1. Digital Fringe Projection Experimental Setup

\subsection{Analysis Method}

In this work, a human hand test subject was tested in two different states, normal positioning of the hand and a slightly deformed finger of the hand. The method adopted for analysis of hand OA are as follow:

1. The fringe patterns generated by $\mathrm{C}++$ coding was shifted three times by $2 \pi / 3$ radians and projected onto the actual hand model in the normal state and deformed state as shown in Figure 2. Image of the hand after fringe projection were recorded with the CCD camera. This process was repeated for five times to ensure each phase shifting algorithms were done in static condition.

2. The three phase shifted recorded images were then applied in the phase mapping process using the UU imaging software to obtain the wrapped phase map [18].

3. A total of 106 digital markers with an equal distance of 50 pixels were plotted onto the wrapped phase map as illustrated in Figure 3. The digital markers were only marked onto the region of actual hand and not the reference plane. Blue markers indicated the centre gridline of the image while the red markers were 
directed to the region of actual finger to be analyzed. The gray scale values and RGB values for each red markers were used as reference.

4. The resultant grayscale and RGB values can be determined from wrapped phase map by conducting deformed state phase shifting algorithms.

5. Comparisons were then made between the normal state and deformed state by constructing a line plot from specified digital markers. If a gray scale or RGB variation happens, pixel tracing of $\mathrm{Y}$-axis will be conducted to detect the equal gray scale value and the new coordinate point location was obtained.

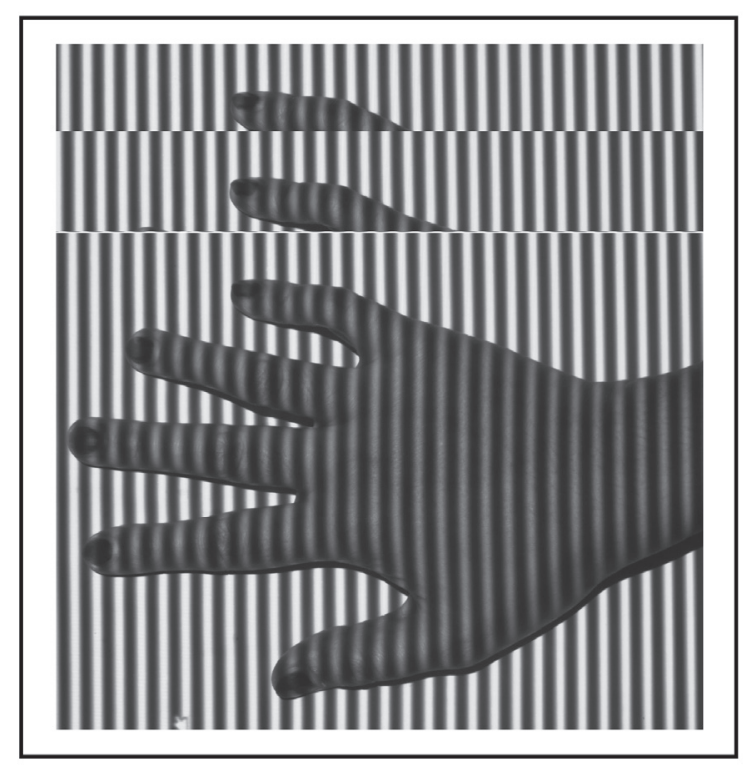

Fig. 2. Three phase shifted images of the hand

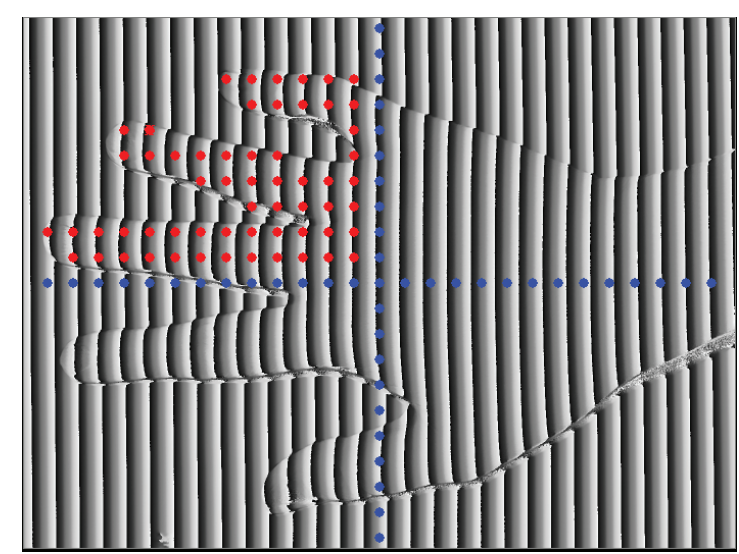

Fig. 3. Phase Map Image of the hand

\section{Results \& Discussion}

The main purpose in this work was to apply digital fringe projection in detecting surface abnormalities of the hand or early stages of hand osteoarthritis. The fringe projection technique adopted the phase shifting technique of the generated fringes. In the analysis, the shifted fringe pattern was projected onto the hand sample in the normal and deformed state whereby a phase map was produced.

Based on the 106 digital markers from the fringe image, the coordinate for each marker was determined from the fringe image. The gray scale value for each coordinate marker was located from the phase map image of the normal hand condition as shown in Figure 4. The area of interest for the hand $\mathrm{OA}$ analysis are the little finger, ring finger and the middle finger demonstrated by the red coordinate markers. Coordinate markers were then plotted for the deformed hand condition with the same area of interest as the normal state phase map image. Comparisons were made for both the gray scale values and an early variation of gray scale value can be observed circled in yellow as shown in Figure 5.

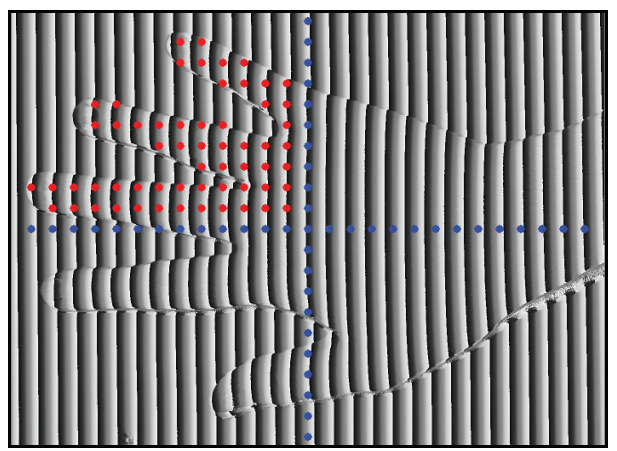

Fig. 4. Coordinate Markers of the normal state of the hand

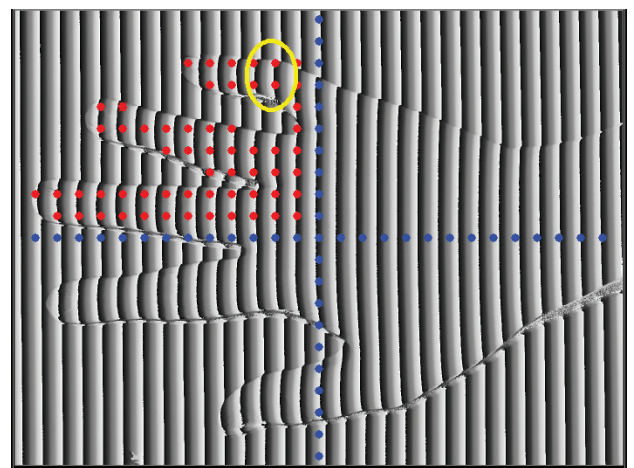

Fig. 5. Coordinate Markers of the deformed state of the hand

Pixel tracing was then conducted and plotted to investigate the surface change of the deformed hand as shown in Figure 6. A total 59 out of the 106 red coordinate markers were plotted onto the wrapped phase map image of the hand test subject shown in Figure 6. The solid blue line signifies the surface plot for the normal hand condition, while the red dashed line signifies the surface plot for the deformed hand. It can be 
seen in that the plotting for both hand conditions are almost identical but for a small number of coordinate location. Based on the phase map image of the deformed hand in Figure 5, the main difference of the pixel coordinate is situated at the little finger location.

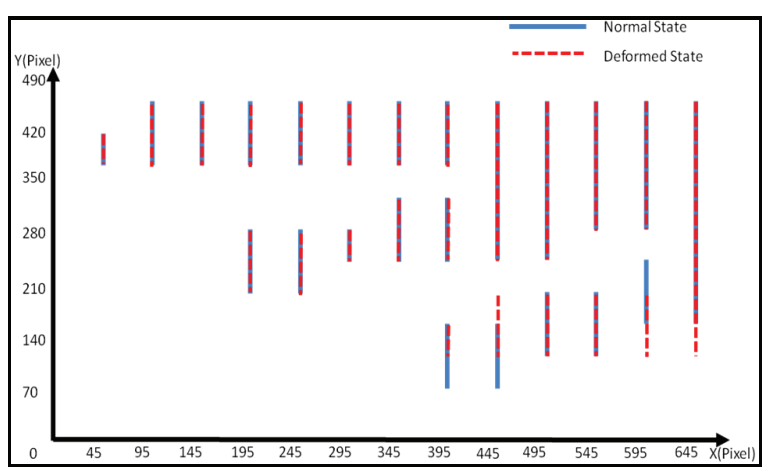

Fig. 6. Hand surface variation plotting of the normal and deformated state of the hand dinate Markers of the normal state of the hand

Evidently the coordinate variation occurs at four main coordinates from the normal hand condition at $\left(\mathrm{x}_{1}, \mathrm{y}_{1}\right)=$ $(395,70),\left(\mathrm{x}_{2}, \mathrm{y}_{2}\right)=(445,70), \quad\left(\mathrm{x}_{3}, \mathrm{y}_{3}\right)=(595,170)$ and $\left(\mathrm{x}_{4}, \mathrm{y}_{4}\right)=(645,190)$. The variation of the deformed coordinates from the normal hand coordinates are shown in Table 1.

Table 1. Pixel tracing comparison of deformed and normal hand state

\begin{tabular}{|c|c|c|}
\hline \multicolumn{3}{|c|}{ Pixel Coordinates } \\
\hline X-axis & Y-Axis (normal) & Y-Axis (deformed) \\
\hline 395 & 70 & 120 \\
\hline 445 & 70 & 120 \\
\hline 595 & 170 & 120 \\
\hline 645 & 190 & 120 \\
\hline
\end{tabular}

The results obtained in this work demonstrate that the application of digital fringe projection can be used in the analysis of hand osteoarthritis or abnormalities. The method applied for hand osteoarthritis detection can be another added technique where its main advantage is that it is a non-contact and free of ionizing radiation compared to the current existing methods. Previous similar technique utilizing digital fringe projection on early breast carcinoma has also been successfully demonstrated [16] and this work has successfully demonstrated another early detection of a medical condition, hand osteoarthritis detection. Extensive analysis will still need to be conducted to ensure a more accurate and efficient application of digital fringe projection on hand osteoarthritis detection.

\section{Conclusions}

The preliminary result of the digital fringe projection system has successfully been proven to be an effective surface measurement for early hand osteoarthritis detection. The phase shift technique and the pixel tracing method have been fully utilized to evaluate the changes of joints and finger deformation. Further improvement can be done by testing the fringe projection system on real patients with osteoarthritis and classify the stages of the patients osteoarthritis which will require a databank of patients medical osteoarthritis history. The fringe shifting and phase mapping method used in this work can also be applied to other medical conditions where physical changes occur.

\section{References}

1. R. W. Moskowitz, Hosp. Pract., 14, 7, pp. 75-80, 85-87, (1979).

2. Doherty, Michael, Hans Bijlsma, Nigel Arden, Nicola Dalbeth, and David J. Hunter, eds. Oxford Textbook of Osteoarthritis and Crystal Arthropathy. Oxford University Press, (2016).

3. R. C. Lawrence et al., Arthritis Rheum., 41, 5, pp. 778-799, (1998).

4. World Health Organization In Summary meeting report, pp. 5-7. (2004).

5. EUMUSC, Eur. Musculoskelet. Heal. Surveill. Infromation Netw., pp. 1-13, (2014).

6. P. Information, "Plain Radiography / X-rays," no. June, (2014).

7. J. F. Holmes and R. Akkinepalli, "Computed Tomography Versus Plain Radiography to Screen for Cervical Spine Injury: A Meta-Analysis," no. May, pp. 902-905, (2005).

8. S. B. Paper, W. Oa, G. Burden, D. Study, E. U. M. States, and U. Nations, 6.12 Osteoarthritis, 12, pp. 6-8, (2010).

9. H. Fuller, R. Fuller, R. Maria, and R. Pereira, Rev. Bras. Ortop. English Ed., 55, 4, pp. 352-362, (2015).

10. H. I. Keen et al., "The development of a preliminary ultrasonographic scoring system for features of hand osteoarthritis," pp. 651-656, (2007).

11. Luebberding, S., Krueger, N., \& Kerscher, M., Dermatologic Surgery, 40, 1, 22-32 (2014).

12. Munera, N., Lora, G. J., \& Garcia-Sucerquia, J., Dyna, 79, 171, 65-73 (2012).

13. Huang, S., Zhang, Z., Zhao, Y., Dai, J., Chen, C., $\mathrm{Xu}, \mathrm{Y} . \mathrm{Xie}, \mathrm{L}$. Optics and Lasers in Engineering, 52, 123-130 (2014). 
14. Rohr, M., \& Schrader, A, In Non Invasive Diagnostic Techniques in Clinical Dermatology, Springer. pp. 55-64) (2014).

15. Saito, M. T., Yoshimura, E. M., Palacios, F. F., Lino, A. C. L., Palacios, F., \& Sousa, M. V. P., SPIE BiOS, 8572, 1-7 (2013).

16. Vairavan, Rajendaran, Vithyacharan Retnasamy, Mukhzeer Mohamad Shahimin, Zaliman Sauli, Lai Siang Leng, Wan Mokhzani Wan Norhaimi, Prema Boshani Retnasamy, Othman Abdullah, and Supap Kirtsaeng. Proc. of SPIE Vol, 10043, pp. 1004314-1. (2017).

17. Retnasamy, V., \& Ratnam, M. MThe International Journal of Advanced Manufacturing Technology, 38(11-12), 1172-1180 (2008).

18. Chen, L., In International Conference on Optics in Precision Engineering and Nanotechnology (icOPEN2013) (p. 87690U-87690U). International Society for Optics and Photonics (2013). 